\title{
BIOLOGIE DES VECTEURS ET TRANSMISSION de Plasmodium falciparum, P. malariae et P. ovale DANS UN VILLAGe DE SAVANE D'AfRIQUE DE L'OUEST (Dielmo, Sénégal)
}

\author{
KONATE L.***, DIAGNE N.*, BRAHIMI K.**, FAYE O.***, LEGROS F.*, ROGIER C.****, PETRARCA V.***** et TRAPE J.-F.*
}

Summary : VeCtORS BIONOMICS AND TRANSMISSION OF PLASMODIUM FALCIPARUM, P. MALARLAE AND P. OVALE IN A SUDAN SAVANNA AREA OF West Africa (Dielmo, Senegal)

From April 1990 to March 1992 a longitudinal entomological study was carried out in Dielmo village, Senegal, an area of Sudan-type savanna. Mosquitoes were sampled by night-bite collections and pyrethrum spray collections. Seven anopheles species were identified: An. gambiae s.s, An. arabiensis, An. funestus, An. pharoensis, An. rufipes, An. squamosus and An. ziemanni. Present throughout the year, An. gambiae s.l. and An. funestus represented more than $98 \%$ of anopheles captured on man. A yearly wave of An. gambiae s.l. was observed in the rainy season and An. funestus was generally more abundant in the dry season. The sporozoite rate was $1.5 \%$ and $1.3 \%$, respectively, for these two species. Sporozoite typing by monoclonal antibodies indicated that the proportion of infected salivary glands was $92.7 \% P$. falciparum, $18.2 \%$ P. malariae and $8.2 \%$ P. ovale. The inoculation rate was calculated to be respectively 111,21 and 8 infective bites per human for $P$. falciparum, $P$. malariae and $P$. ovale during the first year. Transmission was highest in the second year, with respectively 272, 54 and 25 infective bites per human.

KEY WORDS : malaria. Anopheles gambiae s.l. Anopheles funestus, epidemiology. Plasmodium falciparum. Plasmodium malariae. Plasmodium ovale. Senegal.

\section{INTRODUCTION}

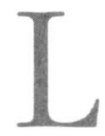

a transmission du paludisme présente d'importantes variations au Sénégal selon les zones bioclimatiques et différents facteurs naturels ou anthropiques. En zone rurale, la transmission varie ainsi d'environ une piqûre infectante par personne par an en zone sahélienne à plusieurs centaines de piqûres infectantes par personne par an en zone soudano-guinéenne (Vercruysse, 1985; Faye, 1987). Dans toutes les régions du pays, des variations saisonnières importantes du niveau de transmission sont obser-

\footnotetext{
* Laboratoire de Paludologie, ORSTOM, B.P. 1386, Dakar, Sénégal

** Laboratoire de Parasitologie Biomédicale, Institut Pasteur, Paris, France

**** Département de Biologie Animale, Université Cheikh Anta Diop, Dakar, Sénégal

***** Service d'Épidémiologie, Institut Pasteur de Dakar, Sénégal

******* Istituto di Parassitologia, Università di Roma "La Sapienza", Italie

Correspondance : Dr J.F. TRAPE, ORSTOM, B.P. 1386, DAKAR Tél : (221) 32 O9 62/321846 - Fax : (221) $321675 / 324307$
}

\section{Résumé}

Une étude entomologique longitudinale a été effectuée à Dielmo, un village situé en zone de savane soudanienne au Sénégal, d'avril 1990 à mars 1992. Les moustiques ont été échantillonnés à partir de captures de nuit sur appâts humains et de pulvérisations intradomicilaires de pyrèthrines. Sept espèces anophèliennes ont été collectées: An. gambiae s.s, An. arabiensis, An. funestus, An pharoensis, An. rufipes, An. squamosus et An. ziemanni. Présents toute l'année, An. gambiae s.l. et An. funestus ont constitué plus de $98 \%$ des anophèles capturés sur homme et ont présenté une nette alternance saisonnière, An. gambiae s.l. présentant un pic annuel en saison des pluies et An. funestus dominant généralement en sai son sèche. L'indice sporozoiltique moyen a été de 1,5\% pour An. gambiae s.l. et de 1,3\% pour An. funestus. L'identification spécifique des sporozoiltes à l'aide d'anticorps monoclonaux a montré que $P$. falciparum, P. malariae et $P$. ovale étaient présents dans respectivement $92,7 \%, 18,2 \%$ et $8,2 \%$ des glandes salivaires infectées. Le taux d'inoculation entomologique pour chacune des trois espèces plasmodiales a été estimé respectivement à 111, 21 et 8 piqûres infectantes par personne la première année d'étude et 272 , 54 et 25 piqûres infectantes par personne la seconde année.

MOTS CLES : paludisme. Anopheles gambiae s.l. Anopheles funestus. épidémiologie. Plasmodium falciparum. Plasmodium malariae. Plasmodium ovale. Sénégal.

vées, avec le plus souvent une interruption complète pendant la majeure partie de la saison sèche (Vercruysse et Jancloes, 1981; Petrarca et al., 1987; Trape et al., 1992; Faye et al., 1992 et 1993).

La présente étude s'inscrit dans le cadre d'un important programme de recherches sur les relations hôtevecteur-parasite et les mécanismes de l'immunité protectrice dans le paludisme, programme débuté en 1990 dans le village de Dielmo (Sine-Saloum) où cette affection est holoendémique (Rogier \& Trape, 1993, Trape et al., 1994). Malgré le nombre important d'études entomologiques effectuées en Afrique tropicale, peu d'entre elles ont été associées à des études parasitologiques, cliniques et séro-immunologiques permettant une analyse approfondie de la relation entre la transmission et les modalités de l'infection dans les populations exposées. L'objectif du présent travail est de préciser la bioécologie des anophèles vecteurs et de mesurer les principaux paramètres entomologiques de la transmission de chaque espèce plasmodiale dans la population étudiée. 


\section{MATÉRIELS ET MÉTHODES}

\section{ZONE D'ÉTUDE}

L e village de Dielmo (13\%45' Nord, $16^{\circ} 25^{\prime}$ ' Ouest) est situé en zone de savane soudanienne, à 280 $\mathrm{km}$ au sud-est de Dakar et à une quinzaine de $\mathrm{km}$ au nord de la frontière gambienne. Une particularité importante de ce village est d'être riverain d'une petite rivière permanente aux rives marécageuses (la Néma), qui procure toute l'année des gîtes larvaires aux anophèles. La pluviométrie est concentrée sur quatre mois, les premières pluies survenant habituellement dans la deuxième moitié de juin et les dernières pluies à la mi-octobre. La pluviométrie annuelle moyenne, qui était d'environ $1000 \mathrm{~mm}$ jusqu'à la fin des années 60 , a diminué de plus de $25 \%$ depuis le début de la période de sècheresse. En 1990 et 1991, années de l'étude, la pluviométrie était respectivement de $635 \mathrm{~mm}$ et $611 \mathrm{~mm}$. La température annuelle moyenne est de $26,5^{\circ} \mathrm{C}$ (minimum : $24^{\circ} \mathrm{C}$ en janvier, maximum: $29^{\circ} \mathrm{C}$ en mai).

Le village compte 250 habitants (sexe ratio $\mathrm{F} / \mathrm{H}$ : 0,98), en majorité d'ethnie Sérère, dont $47 \%$ d'enfants de moins de 15 ans. La population est répartie entre le village principal de Dielmo (20 concessions et 195 habitants) et le petit hameau de Santhe-Mouride (9) concessions et 55 habitants), distant de 300 mètres. La majorité des habitations $(62 / 87=71 \%)$ est de construction traditionnelle avec murs en banco et toit de chaume, les autres $(25 / 87=29 \%)$ ont un toit en tôle ondulée non jointif avec le haut des murs. La population active est constituée exclusivement d'agriculteurs sédentaires. De grands manguiers ont été plantés autour des habitations tandis que la savane arborée environnante a été presque totalement défrichée pour les cultures. Le mil est cultivé avec l'arachide pendant la saison des pluies. En saison sèche, le maraîchage, un peu de riziculture et la cueillette des mangues constituent les seules activités agricoles. Les animaux domestiques (environ 40 bovidés, 300 ovins et caprins, 20 équidés et de la volaille) sont gardés pendant la nuit dans des enclos et basses-cours, à proximité immédiate des habitations humaines.

\section{Méthodes entomologiques}

Les captures de nuit sur appâts humains ont été effectuées à Dielmo-village sur une période de deux ans, à l'intérieur et à l'extérieur de deux maisons considérées comme représentatives du village à l'issue d'une série préliminaire de captures de la faune matinale résiduelle, la première semaine de chaque mois (deux séances mensuelles d'avril à juin 1990, trois séances mensuelles de juillet 1990 à mars 1992). Les moustiques étaient capturés de $21 \mathrm{~h}$ à $7 \mathrm{~h}$ du matin par quatre équipes de deux captureurs, deux à l'intérieur et deux à l'extérieur des habitations, chaque captureur travaillant et se reposant alternativement pendant une heure. À la fin de chaque heure, les anophèles étaient collectés et disséqués pour la recherche de sporozoïtes dans leurs glandes salivaires et la détermination de l'état de parturité par l'examen des trachéoles ovariens (Detinova, 1963). En cas de présence de sporozoïtes, ceux-ci étaient séchés sur lame puis identifiés par immunofluorescence en utilisant des anticorps monoclonaux spécifiques de P. falciparum, P. malariae et $P$. ovale selon une méthode séquentielle décrite par ailleurs (Trape et al., 1994).

Les captures intra-domiciliaires après pulvérisation de pyrèthrines ont été entreprises sur une période d'un an, de mai 1990 à avril 1991. Elles étaient effectuées vers $7 \mathrm{~h}$ du matin la première semaine de chaque mois, dans 20 chambres de maisons différentes, pour moitié à Dielmo-village et à Santhe-Mouride. Après identification, les femelles d'anophèles étaient classées selon l'état de réplétion de leur abdomen (à jeun, gorgées, semi-gravides, gravides). L'origine du repas de sang a été déterminée par ELISA d'après la méthode de Beier et al. (1988a) sur un échantillon de 175 femelles d'An. gambiae s.l. et d'An. funestus collectées dans des habitations, des greniers et des étables. Un sondage cytotaxonomique du complexe gambiae (méthode de Coluzzi et al., 1979) a été effectué chez un échantillon de 56 femelles semi-gravides endophiles d'An. gambiae s.l. collectées vers $7 \mathrm{~h}$ du matin en saison sèche (janvier et févier 1991) et en saison des pluies (septembre 1991).

\section{RÉSULTATS}

\section{FAUNE ANOPHÉLIENNE}

L ors des captures de nuit, 18585 Culicidae femelles dont 11369 femelles d'anophèles (61,2\%) ont été capturées pour un total de 276 hommes-nuits. Il s'agissait de : 7144 An. gambiae s.l (62,8 \%), 4040 An. funestus (35,6 \%), 140 An. pharoensis (1,2\%), 19 An. rufipes $(0,2 \%), 15$ An. ziemanni $(0,1 \%)$ et 11 An. squamosus $(0,1 \%)$.

Les pulvérisations intra-domicilaires de pyrèthrines ont permis la collecte de 17546 Culicidae pour un total de 240 chambres étudiées. Il s'agissait de 6132 mâles et de 11414 femelles dont 9236 femelles d'anophèles (81 \%). La répartition par espèces est indiquée sur le tableau I. Les espèces les plus fréquemment récoltées par pulvérisation ont été $A n$. gambiae s.l. (59\%), An. rufipes (28\%) et An. funes- 
tus $(13 \%)$. Leur répartition était différente dans les deux localités $(\mathrm{P}<0,001)$. Il a été récolté quatre fois plus d'An. rufipes à Dielmo-village qu'à SantheMouride où An. gambiae s.l. et An. funestus ont également été moins abondants.

L'examen des chromosomes polytènes des cellules trophocytaires des ovocytes chez 56 femelles semigravides d'An. gambiae s.l. a permis d'identifier 48 An. gambiae s.s. (IC 95\%: $76 \%-95 \%$ ) et 8 An. arabiensis (IC $95 \%: 5 \%-23 \%$ ). Chacune de ces deux espèces a été observée en saison sèche comme en saison des pluies. Les principales inversions chromosomiques d'An. gambiae étaient 2Rb, 2Rd et 2La. Les arrangements chromosomiques indiquaient la présence des cytotypes Forêt, Savane et Bissau.

\section{Bió́COLOGIE}

Variations mensuelles des densités anophèliennes

An. gambiae s.l. et An. funestus ont été capturés sur homme pendant chacun des 24 mois de l'étude (fig. 1). Le nombre de piqûres d'An. gambiae s.l. par personne et par nuit (PHN) a été maximal entre juillet et septembre en saison des pluies, et minimal entre novembre et avril en saison sèche. La densité agressive moyenne annuelle d'An. funestus a été près de cinq fois plus faible pendant la première année (5 PHN) que pendant la deuxième année d'étude (23 PHN) où il a été noté deux maxima : le premier, juste avant la saison des pluies (48 PHN en juin 1991) et le second, en milieu de saison sèche (41 PHN en février 1992).

Les variations mensuelles du nombre d'An. gambiae s.l. et d'An. funestus par pièce, récoltés après pulvérisations (fig. 2), ont été identiques à celles des captures sur homme. Le nombre maximum de femelles d'An. rufipes par pièce a été observé en juillet.

\section{Cycle d'agressivité}

Les variations horaires du taux d'agressivité d'An. gambiae s.l. et d'An. funestus sont présentées sur la figure 3. Pour ces deux espèces l'agressivité maximale a été observée dans la deuxième moitié de la nuit.

\section{Taux d'endophagie}

Le taux moyen d'endophagie (rapport du nombre moyen de moustiques se gorgeant à l'intérieur sur le total capturé) a été de $53 \%$ (IC $95 \%$ : 52-54\%) pour An. gambiae s.l. et de $59 \%$ (IC $95 \%$ : 58-60\%) pour An. funestus ( $\mathrm{p}<0,001)$. Au cours de la nuit (fig. 3), ce taux a varié significativement en fonction de la tranche horaire pour chacune de ces deux espèces (respectivement $p<0,0001$ et $p<0,001)$. Les valeurs les plus faibles ont été observées en début et en fin de nuit.

État physiologique des femelles endophiles

Parmi les femelles d'An. gambiae s.l. capturées en faune matinale résiduelle, 228 étaient à jeun (4,2\%), 3126 étaient gorgées $(57,4 \%$ ), 493 étaient semi-gravides $(9,0 \%)$ et 1602 étaient gravides (29,4\%). Pour An. funestus, les valeurs correspondantes ont été respectivement de $91(7,4 \%), 699(56,6 \%), 232(18,8 \%)$ et $212(17,2 \%)$. Les différences observées entre les deux espèces sont significatives $(p<0,001)$.

\section{Préférences trophiques}

Pour An. gambiae s.l., l'origine du repas de sang a été déterminée chez 109 femelles gorgées collectées dans les habitations humaines, chez 34 femelles collectées dans les greniers et chez 34 autres femelles collectées dans les étables à ovins-caprins. Dans les habitations humaines, $75 \%$ des femelles s'étaient gorgées sur homme, $24 \%$ sur bovidés et $1 \%$ sur ovidés ou capridés. Dans les étables, les proportions correspondantes ont été respectivement de $35 \%, 55 \%$ et $10 \%$. Dans les greniers, $45 \%$ des femelles d'An. gambiae s.l. s'étaient gorgées sur homme et $55 \%$ sur bovidés.

Pour les 144 An. funestus récoltés dans les habitations humaines, $92 \%$ s'étaient gorgés sur homme et $5 \%$ sur bovidés. La différence d'anthropophilie entre $A n$. funestus et An. gambiae s.l. pour les femelles capturées dans les habitations est significative $(p<0,001)$.

\section{Taux de parturité}

La valeur moyenne du taux de parturité (TP) a été de $72 \%$ pour An. gambiae s.l. (Intérieur : 74\%; Extérieur : $71 \% ; \mathrm{p}<0,05)$ et $76 \%$ pour An. funestus (Intérieur : $75 \%$; Extérieur : $76 \%$; n.s.). Le TP de ces deux espèces a varié significativement en fonction de la tranche horaire $(\mathrm{p}<0,001)$, avec un minimum en début de nuit (fig. 4). Les variations mensuelles du TP d'An. gambiae s.l. et d'An. funestus sont indiquées

\begin{tabular}{|c|c|c|c|c|c|c|c|c|c|}
\hline \multirow[b]{2}{*}{ Localités } & \multirow{2}{*}{$\begin{array}{l}\mathrm{Nb} \text {. de } \\
\text { séances }\end{array}$} & \multirow{2}{*}{$\begin{array}{l}\text { Nb. pièces } \\
\text { visitées }\end{array}$} & \multicolumn{6}{|c|}{ Nombre de femelles d'anophèles récoltées (\%) } & \multirow{2}{*}{$\begin{array}{c}\text { Total } \\
\text { anophèles }\end{array}$} \\
\hline & & & $\begin{array}{c}\text { An. } \\
\text { gambiae s.l. }\end{array}$ & An. funestus & An. rufipes & $\begin{array}{c}\text { An. } \\
\text { pharoensis }\end{array}$ & An. ziemanni & $\begin{array}{c}\text { An. } \\
\text { squamosus }\end{array}$ & \\
\hline Dielmo-village & 24 & 120 & $3082(52,8)$ & $760(13,0)$ & $1994(34,1)$ & $3(0,05)$ & - & $3(0,05)$ & 5842 \\
\hline Santhe-Mouride & 24 & 120 & $2367(69,8)$ & $474(13,9)$ & $549(16,2)$ & - & $1(0,02)$ & $3(0,08)$ & 3394 \\
\hline Total & 48 & 240 & $5449(58,9)$ & $1234(13,4)$ & $2543(27,6)$ & $3(0,03)$ & $1(0,01)$ & $6(0,06)$ & 9236 \\
\hline
\end{tabular}

Tableau I - Résultats des collectes de la faune matinale résiduelle, mai 1990-avril 1991 

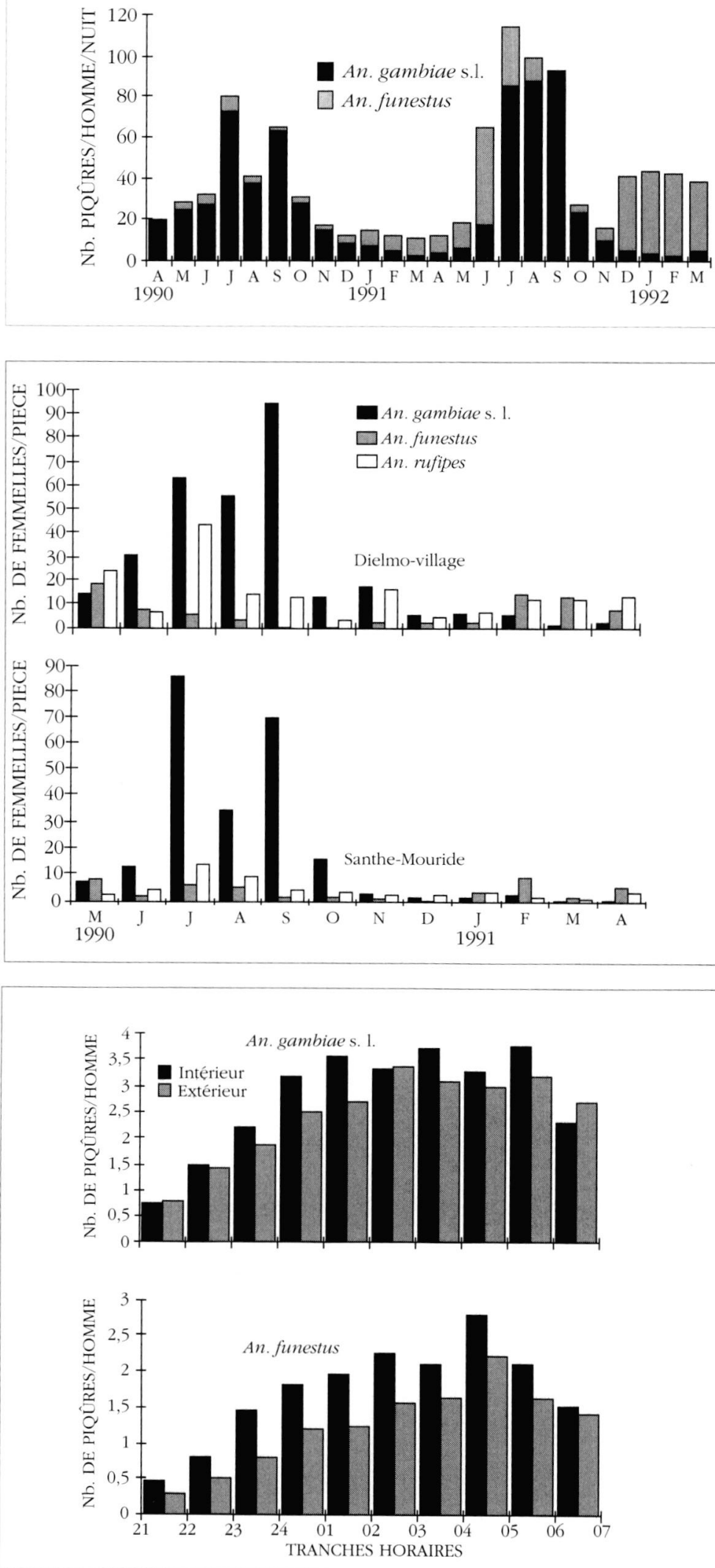

Fig. 1. - Variations mensuelles des densités agressives d'An. gambiae s.l. et d' $A n$. funestus. Dielmo, avril 1990-mars 1992.

Fig. 2. - Variations mensuelles des densités d'An. gambiae s.l. , d'An. funestus et d'An. rufipes par pièce d'habitation. Dielmo-village et Santhe Mouride, mai 1990-avril 1991.

Fig. 3. - Taux d'agressivité horaire d'An. gambiae s.l. et d'An. funestus à l'intérieur et à l'extérieur des habitations. Dielmo, avril 1990mars 1992. 


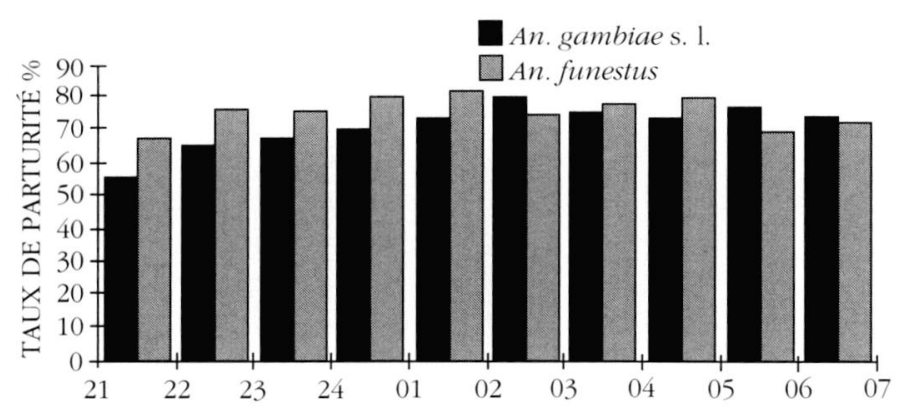

Fig. 4. - Variations horaires du taux de parturité d'An. gambiae s.l. et d'An. funestus. Dielmo, avril 1990mars 1992.

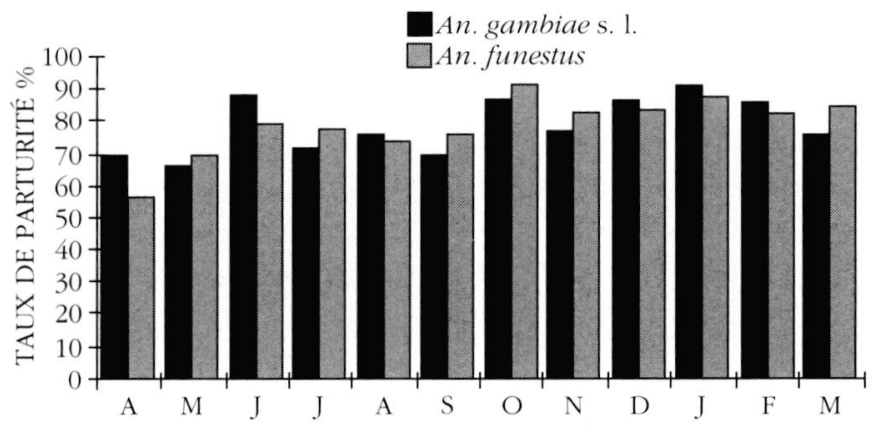

Fig. 5. - Variations mensuelles du taux de parturité d'An. gambiae s.l. et d'An. funestus. Dielmo, avril 1990-mars 1992.

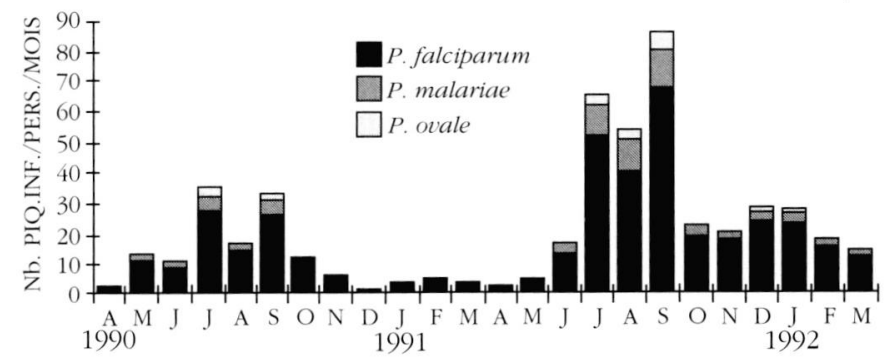

Fig. 6. - Variations mensuelles du taux d'inoculation entomologique de P. falciparum, P. malariae et P. ovale. Dielmo, avril 1990-mars 1992. 
sur la Figure 5. Le TP moyen d'An. gambiae s.l. a été de $72 \%$ en saison des pluies et de $73 \%$ en saison sèche (n.s.). Pour An. funestus, les valeurs correspondantes ont été respectivement de $74 \%$ et $76 \%$ en saison des pluies et en saison sèche (n.s.).

\section{TRANSMISSION DES PLASMODIES HUMAINES}

Indices sporozoïtiques

L'indice sporozoïtique a été de $1,5 \%$ (IC $95 \%$ : 1,1$1,7 \%$ ) pour $A n$. gambiae s.l. (Intérieur : 1,4\%; Extérieur : 1,5\%; n.s.) et de 1,3\% (IC $95 \%: 0,9-$ $1,6 \%$ ) pour An. funestus (Intérieur : 1,3\%; Extérieur : $1,2 \% ;$ n.s.) (Tableau II). L'indice sporozoïtique moyen (An. gambiae s.l. et An. funestus) a été significativement plus élevé la deuxième année d'étude que pendant la première année (1,6\% vs. $1,0 \%, \mathrm{p}<0,05)$ et en saison des pluies qu'en saison sèche $(1,7 \%$ vs. $1,0 \%, \mathrm{p}<0,01)$.

Identification spécifique des sporozoïtes

L'identification des espèces plasmodiales a été effectuée sur 110 des 116 (95\%) lames de sporozoïtes testées (présence de sporozoïtes non fluorescents dans trois cas, détachement des sporozoïtes dans trois autres cas). Les sporozoïtes de ces 110 glandes salivaires infectées appartenaient à une seule espèce plasmodiale dans $86 \%$ des cas. $8 \%$ des glandes salivaires contenaient deux espèces plasmodiales tandis que $6 \%$ contenaient trois espèces plasmodiales. La proportion relative de chaque espèce plasmodiale dans les infections de glandes salivaires a été respectivement de $92,7 \%, 18,2 \%$ et de $8,2 \%$ pour $P$. falciparum, $P$. malariae et $P$. ovale. Ces trois espèces plasmodiales ont été trouvées seules ou associées chez chacun des vecteurs.

\section{Taux d'inoculation entomologique}

Les taux mensuels d'inoculation entomologique ont été calculés pour chaque espèce vectrice à partir du produit de la densité agressive et de la moyenne mobile de l'indice sporozoïtique (moyenne du mois considéré, du mois précédent et du mois suivant) Ainsi, pendant la durée de l'étude, le nombre de piqûres potentiellement infectantes par personne a été de 412 dont 294 (71\%) durant la deuxième année. Pour An. gambiae s.l., le taux d'inoculation entomologique a été de 285 piqûres infectantes par personne (première année : 94; deuxième année : 191). An. gambiae s.l. à toujours assuré l'essentiel de la transmission en saison des pluies, avec un maximum de 72 piqûres infectantes par personne par mois en septembre 1991. Pour An. funestus, le taux d'inoculation entomologique a été de 127 piqûres infectantes par personne (première année : 24; deuxième année : 103). Il a atteint un maximum de 19 piqûres infectantes par personne par mois en décembre 1991, en milieu de saison sèche.

Les taux d'inoculation entomologique mensuels de $P$. falciparum, $P$. malariae et $P$. ovale, estimés à partir de la proportion de l'espèce plasmodiale considérée dans la totalité des infections de glandes salivaires identifiées au cours de l'étude, sont présentés sur la figure 6. La première année d'étude, les taux d'inoculation ont été estimés à respectivement 111, 21 et 8 piqûres infectantes par personne pour $P$. falciparum, $P$. malariae et $P$. ovale. Ils ont été plus élevés la deuxième année avec respectivement 272,54 et 25 piqûres infectantes par personne.

Analyse épidémiologique de la transmission

Les principaux paramètres entomologiques de la transmission du paludisme à Dielmo sont indiqués sur le tableau III. La durée de la sporogonie (n) à Dielmo a été calculée à partir de la formule de Moskhovsky (Detinova, 1963) pour une température moyenne annuelle de $26,5^{\circ} \mathrm{C}$. La durée du cycle gonotrophique d'An. gambiae s.l. et d'An. funestus, non étudiée à Dielmo, a été estimée à deux jours à partir des données de la littérature (Gillies \& De Meillon, 1968).

\begin{tabular}{|c|c|c|c|c|c|c|c|c|}
\hline \multirow{2}{*}{\multicolumn{2}{|c|}{ Espèces }} & \multicolumn{3}{|c|}{ Première année } & \multicolumn{3}{|c|}{ Deuxième année } & \multirow{3}{*}{$\begin{array}{c}\text { Total } \\
5681 \\
83 \\
1,46\end{array}$} \\
\hline & & \multirow{2}{*}{$\begin{array}{c}\text { S. pluies } \\
1784 \\
21 \\
1,18\end{array}$} & \multirow{2}{*}{$\begin{array}{c}\text { S. sèche } \\
959 \\
5 \\
0,52\end{array}$} & \multirow{2}{*}{$\begin{array}{c}\text { Total } \\
2743 \\
26 \\
0,95\end{array}$} & \multirow{2}{*}{$\begin{array}{c}\text { S. pluies } \\
2395 \\
51 \\
2,13\end{array}$} & \multirow{2}{*}{$\begin{array}{c}\text { S. sèche } \\
543 \\
6 \\
1,10\end{array}$} & \multirow{2}{*}{$\begin{array}{c}\text { Total } \\
2938 \\
57 \\
1,94\end{array}$} & \\
\hline A. gambiae s.l. & $\begin{array}{c}\text { Disséqué } \\
\text { Gl+ } \\
\text { s (\%) }\end{array}$ & & & & & & & \\
\hline A. funestus & $\begin{array}{c}\text { Disséqué } \\
\text { Gl+ } \\
\text { s }(\%)\end{array}$ & $\begin{array}{c}167 \\
2 \\
1,19\end{array}$ & $\begin{array}{c}413 \\
6 \\
1,45\end{array}$ & $\begin{array}{c}580 \\
8 \\
1,38\end{array}$ & $\begin{array}{c}352 \\
8 \\
2,27\end{array}$ & $\begin{array}{c}2550 \\
28 \\
1,09\end{array}$ & $\begin{array}{c}2902 \\
36 \\
1,24\end{array}$ & $\begin{array}{c}3582 \\
44 \\
1,26\end{array}$ \\
\hline
\end{tabular}

Tableau II - Variations saisonnières de l'indice sporozoïtique (s) d'An. gambiae s.l. et d'An. funestus. Dielmo, avril 1990-mars 1992. Gl+ = nombre de moustiques trouvés porteurs de sporozoïtes. 
Nombre moyen de piqûres par homme par nuit ( $m a$ )

Proportion de femelles pares (A)

Rythme quotidien de piqûres sur homme (a)

Durée du cycle gonotrophique $(x)$ en jours

Taux quotidien de survie $(\mathrm{p}=\sqrt[\times]{\mathrm{A}})$

Durée du cycle extrinsèque $(n)$ de $P$. falciparum à $26,5^{\circ} \mathrm{C}$

Taux de survie après $n$ jours $\left(\mathrm{p}^{n}\right)$

Espérance de vie $\left(1 /-\log _{e} p\right)$ en jours

Espérance de vie infectante $\left(\mathrm{p}^{n} /-\log _{e} p\right)$ en jours

Indice de stabilité $\left(a /-\log _{e} p\right)$

Capacité vectorielle $\left(\mathrm{C}=m a^{2} \mathrm{p}^{n} /-\log _{e} p\right)$

$\begin{array}{cc}25,88 & 14,64 \\ 0,72 & 0,76 \\ 0,32 & 0,46 \\ 2 & 2 \\ 0,849 & 0,872 \\ 10,6 & 10,6 \\ 0,176 & 0,234 \\ 6,10 & 7,30 \\ 1,07 & 1,70 \\ 1,95 & 3,36 \\ 8,84 & 11,46\end{array}$

Tableau III - Paramètres entomologiques de la transmission du paludisme à Dielmo, avril 1990-mars 1992.

\section{DISCUSSION}

S ur les vingt espèces d'anophèles dont la présence est connue au Sénégal (Diagne et al., sous presse), sept espèces ont été identifiées au cours de cette étude : An. gambiae s.s., An. arabiensis, An. funestus, An. rufipes, An. pharoensis, An. ziemanni et An. squamosus. Parmi elles, seuls $A n$. gambiae s.s., An. arabiensis et An. funestus interviennent dans la transmission du paludisme à Dielmo. Le rôle de vecteur majeur du paludisme d'An. gambiae s.l. est constamment observé au Sénégal (Vercruysse et Jancloes, 1981; Vercruysse et al., 1985; Trape et al., 1992; Faye et al., 1993). Sur les trois espèces de ce complexe, on remarque l'absence de An. melas malgré la proximité relative (environ $7 \mathrm{~km}$ ) des zones de mangroves où cette espèce est abondante (Bryan et al., 1982). An. funestus était autrefois présent dans de nombreuses localités du centre et du sud du Sénégal (Hamon et al., 1955; 1956a; 1956b), mais est actuellement beaucoup plus rare, certainement en raison de l'impact de la persistance de la sècheresse sur ses gites larvaires potentiels. Dans le cas de Dielmo, le maintien d'une petite rivière permanente malgré un important déficit pluviométrique permet à cette espèce de conserver un rôle essentiel dans la transmission du paludisme pendant la saison sèche, selon des modalités bien connues en zone de savane soudanienne d'Afrique de l'Ouest (Gillies \& De Meillon, 1968; Brengues \& Coz, 1973). Bien que An. pharoensis soit un vecteur secondaire possible du paludisme dans certaines régions du Sénégal où cette espèce est très abondante (Carrara et al., 1990), il ne joue aucun rôle dans la transmission à Dielmo. L'abondance d'An. rufipes dans les collectes de faune matinale résiduelle et son absence quasi totale lors des captures nocturnes sur homme, traduisent sa stricte zoophilie à Dielmo, contrairement à ce qui a parfois été mentionné dans d'autres régions d'Afrique (Gillies \& De Meillon, 1968).
L'identification spécifique des sporozoïtes a montré qu'en toute saison An. gambiae s.l. et An. funestus sont souvent simultanément infectés par deux ou trois espèces de Plasmodium. La méthode utilisée, qui permet une visualisation directe des sporozoïtes, a constamment mis en évidence des différences de morphologie entre les sporozoïtes des espèces plasmodiales associées, confirmant ainsi la spécificité des résultats obtenus. Peu d'études en Afrique ont comporté une identification des sporozoïtes chez les vecteurs, et toutes ont utilisé la technique ELISA qui ne permet pas la visualisation des sporozoïtes (Beier et al., 1988b; Boudin et al., 1991; Fontenille et al., 1992). La proportion relative de chaque espèce plasmodiale, de même que celle des associations d'espèces dans les infections de glandes salivaires, ont été comparables à celles rapportées par Beier et al., (1988b) en zone holoendémique au Kenya.

L'existence à Dielmo d'une petite rivière permanente y entretient un anophèlisme continu. La densité anophèlienne moyenne a été plus élevée la deuxième année d'étude, en relation directe avec l'augmentation de la densité d'An. funestus. Nous n'avons pas d'explication pour les deux pics présentés par cette espèce à des périodes différentes de l'année, le premier en juin 1991 et le second de décembre 1991 à mars 1992, pics qui n'ont pas été retrouvés d'une année à l'autre malgré des profils climatiques similaires. Pour An. gambiae, une nette augmentation de la densité vectorielle à toujours précédé les premières pluies. Ceci est probablement en relation avec l'augmentation de la température, qui est maximum à partir d'avril ou mai, et surtout l'augmentation de l'humidité associée à la remontée du front intertropical, qui intervient environ un mois avant les premières pluies.

Les résultats de cette étude montrent que les habitants de Dielmo sont soumis en permanence à une transmission du paludisme élevée. Dès leurs premiers mois de vie, seule une très faible proportion des ino- 
culations reçues par les habitants de Dielmo se traduit par des accès cliniques (Rogier \& Trape, 1993; Trape et al., sous presse). Le choix de Dielmo apparait ainsi adapté à l'étude des déterminants de la morbidité palustre et des mécanismes de l'immunité protectrice en situation d'holoendémicité palustre. De plus, la proximité immédiate d'autres villages où la transmission est interrompue la majeure partie de l'année, devrait permettre d'approfondir la compréhension des facteurs liés à la transmission en incluant dans un même protocole d'étude des populations ne différant que par leurs modalités d'exposition au paludisme.

\section{REMERCIEMENTS}

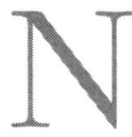

ous remercions Abdoulaye Diop, Moussa Diagne (Service de Lutte Antiparasitaire de Thiès), Hilaire Bouganali et Charles Bouganali (ORSTOM) pour leur assistance technique et les habitants de Dielmo qui ont assuré la capture des moustiques. Nous exprimons également nos plus vifs remerciements à Luiz Pereira da Silva, Pierre Druilhe et Mario Coluzzi pour l'appui apporté à cette étude.

Ce travail a bénéficié d'un soutien financier du Ministère de la Recherche et de l'Enseignement Supérieur et du Ministère de la Coopération et du Développement.

\section{BIBLIOGRAPHIE}

Beier J.C., Perkins P.V., Wirtz R.A., Koros J., Digg D., Gargan T.P. \& Koech D.K. Bloodmeal identification by direct enzyme-linked immunosorbent assay (ELISA), tested on Anopheles (Diptera: Culicidae) in Kenya. Journal of Medical Entomology, 1988a, 25, 9-16.

Beier M.S., Schwartz I.K., Beier J.C., Perkins P.V., Onyango F., Koros J.K., Campbell G.H., Andrysiak P.M. \& Brandling-BennetT A.D. Identification of malaria species by ELISA in sporozoite and oocyst infected Anopheles from western Kenya. American Journal of Tropical Medicine and Hygiene, 1988b, 39, 323-327.

Boudin C., Robert V., Verhave J.P., Carnevale P. \& Ambroise-Thomas P. Plasmodium falciparum and $P$. malariae epidemiology in a West African village. Bulletin of the WHO, 1991, 69, 199-205.

Brengues J. \& Coz J. Quelques aspects fondamentaux de la biologie d'Anopheles gambiae Giles (sp. A) et d'Anopheles funestus Giles, en zone de savane humide d'Afrique de l'Ouest. Cahiers ORSTOM, Série Entomologie Médicale et Parasitologie, 1973, 11, 107-126

Bryan J.H., Di Deco M.A., Petrarca V. \& Coluzzi M. Inversion polymorphism and incipient speciation in Anopheles gambiae s.str. in The Gambia, West Africa. Genetica, 1982, 59, 167-176.
Carrara G.C., Petrarca V., Niang M. \& Coluzzi M. Anopheles pharoensis and transmission of Plasmodium falciparum in the Senegal River delta, West Africa. Medical and Veterinary Entomology, 1990, 4, 421-424.

Coluzzi M., Sabatini A., Petrarca V. \& Di Deco M.A. Chromosomal differentiation and adaptation to human environments in the Anopheles gambiae complex. Transactions of the Royal Society of Tropical Medicine and Hygiene, 1979, 73, 483-497.

Detinova T.S. Méthodes à appliquer pour classer par groupes d'âge les diptères présentant une importance médicale. OMS, Monographie n 47, 1963, 220 p.

Diagne N., Fontenille D., Konate L., Faye O., Legros F., Lamizana M.T., Molez J.F. \& Trape J.F. Les Anophèles du Sénégal. Bulletin de la Société de Pathologie Expérimentale, sous presse.

FAyE O. Contribution à l'étude des Anophelinae (Diptera culicidae) et la transmission du paludisme dans la zone du barrage anti-sel de Bignona, Ziguinchor (Sénégal). Thèse de $3^{\mathrm{e}}$ cycle, Université Cheikh Anta Diop, Dakar, $1987,217 \mathrm{p}$.

Faye O., Diallo S., Gaye O. \& Mouchet J. Évaluation de l'efficacité du Fenitrothion (Sumithion ${ }^{\circledR}$ PM 40) sur la densité du vecteur et la prévalence du paludisme à Pout (Thiès, Sénégal). Annales de la Société Belge de Médecine Tropicale, 1992, 72, 103-112.

Faye O., Fontenille D., Hervé J.P., Diack P.A., Diallo S. \& Mouchet J. Le paludisme en zone sahélienne du Sénégal. 1. Données entomologiques sur la transmission. Annales de la Société Belge de Médecine Tropicale, 1993, 73, 2130 .

Fontenille D., Lepers J.P., Coluzzi M., Campdell G.H., Rakotoarivony I. \& Coulanges P. Malaria transmission and vector biology on Sainte Marie Island, Madagascar. Journal of Medical Entomology, 1992, 29, 197-202.

Gillies M.T. \& De Meillon B. The Anophelinae of Africa South of the Sahara (Ethiopian zoogeographical region). Publications of the South African Institute for Medical Research, 1968, n 54, 343 p.

Hamon J., Abonnenc E. \& Noel E. Contribution à l'étude des culicidés de l'ouest du Sénégal. Annales de Parasitologie Humaine et Comparée, 1955, 3, 277-308.

Hamon J., Adam J.P. \& Grjebine A. Observations sur la répartition et le comportement des anophèles d'Afrique Équatoriale Française, du Cameroun et de l'Afrique Occidentale. Bulletin O.M.S., 1956a, 15, 549-591.

Hamon J., Devemy P., Rickenbach A. \& Gruchet G. Contibution à l'étude des moustiques de la Casamance. Annales de Parasitologie Humaine et Comparée, 1956b, 31, 607-618.

Petrarca V., Vercruysse J. \& Coluzzi M. Observations on the Anopheles gambiae complex in the Senegal River Basin, West Africa. Medical and Veterinary Entomology, 1987, 1, 303-312.

Rogier C. \& Trape J.F. Malaria attacks in children exposed to high transmission: who is protected? Transactions of the Royal Society of Tropical Medicine and Hygiene, 1993, 87, 245-246. 
Trape J.F., Lefebvre-Zante E., Legros F., Ndiaye G., Bouganali H., Druilhe P. \& Salem G. Vector density gradients and the epidemiology of urban malaria in Dakar, Senegal. American Journal of Tropical Medicine and Hygiene, 1992, 47, 181-189.

Trape J. F., Rogier C., Konate L., Diagne N., Bouganali H., Canque B., Legros F., Badji A., Nitaye G., Ndiaye P., Brahimi K., Faye O., Druilhe P. \& Pereira da Silva L. The Dielmo project. A longitudinal study of natural malaria infection and the mechanisms of protective immunity in a community living in a holoendemic area of Senegal. American Journal of Tropical Medicine and Hygiene, 1994, 51, 123-137.

Vercruysse J. Étude entomologique sur la transmission du paludisme humain dans le bassin du fleuve Sénégal (Sénégal). Annales de la Société Belge de Médecine Tropicale, 1985, 65 (suppl. 2), 171-179.

Vercruysse J. \& Jancloes M. Étude entomologique sur la transmission du paludisme humain dans la zone urbaine de Pikine (Sénégal). Cahiers ORSTOM, Série Entomologie Médicale et Parasitologie, 1981, 19, 165-178.

Accepté le 30 septembre 1994 